\title{
Simulation Studies of High-Field EPR Spectra of Spin-Labeled Lipids in Membranes
}

\author{
V. A. Livshits and D. Marsh \\ Abteilung Spektroskopie, Max-Planck-Institut für biophysikalische Chemie, D-37070 Göttingen, Germany; and \\ Centre of Photochemistry, Russian Academy of Sciences, 117427 Moscow, Russian Federation
}

Received December 17, 1999; revised June 5, 2000

The high-field (i.e., $94 \mathrm{GHz}$ ) membrane EPR spectra of lipids spin labeled in their fatty acid chains have been simulated by using two limiting motional models. The aim was to identify the dynamic origin of the residual $\left(g_{x x}-g_{y y}\right)$ anisotropy observed in the nonaxial EPR spectra of cholesterol-containing membranes. It is concluded that the residual spectral anisotropy arises from inplane ordering of the lipid chains by cholesterol. The partial averaging of the $\left(g_{x x}-g_{y y}\right)$ anisotropy was best described by restricted axial rotation with a frequency in the region of $\tau_{\mathbf{R} \|}^{-1} \sim$ $0.5-1 \times 10^{9} \mathrm{~s}^{-1}$. Simulations for slower axial rotation of unrestricted amplitude produced less satisfactory fits. In phospholipid membranes not containing cholesterol, the nonaxial anisotropy is completely averaged in the fluid phase and substantially reduced even in the gel phase. The unrestricted axial rotation in the gel phase is of comparable frequency to that of the limited axial rotation in the liquid-ordered phase of membranes containing cholesterol. These results on in-plane ordering by cholesterol in the liquid-ordered phase could be significant for current proposals regarding domain formation in cellular membranes. $\bigcirc 2000$ Academic Press

\section{INTRODUCTION}

With the advent of a new generation of commercial highfield EPR spectrometers, simulation of the corresponding spectra from nitroxide spin labels in biological systems becomes particularly important and timely. Unlike the situation for normal spin-label EPR studies at microwave frequencies in the 9-GHz region, at higher fields and operating frequencies the anisotropy of the Zeeman interaction becomes increasingly important relative to that of the ${ }^{14} \mathrm{~N}$-hyperfine stucture. At microwave frequencies in the region of $94 \mathrm{GHz}$, the Zeeman anisotropy dominates over the hyperfine anisotropy, and all three canonical positions corresponding to the $g_{x x}, g_{y y}$, and $g_{z z}$ elements of the $g$-tensor may be resolved in the high-field spectra from static samples. The overwhelming advantage is that nonaxial $x-y$ molecular ordering and axial rotations about the spin-label $z$-axis are now amenable to study, which is not possible at the lower operating frequencies of standard spin-label EPR, because the ${ }^{14} \mathrm{~N}$-hyperfine tensor is completely axial. Importantly, compared with EPR spectra at considerably higher fields, the nonaxial $g$-value anisotropy at $94 \mathrm{GHz}$ corresponds to relatively slow rotational motions $\left[\left(g_{x x}-\right.\right.$ $\left.\left.g_{y y}\right) \beta \mathrm{H} / \hbar \sim 8 \times 10^{8} \mathrm{~s}^{-1}\right]$, comparable to those corresponding to the axial ${ }^{14} \mathrm{~N}$-hyperfine anisotropy $\left[\left(A_{z z}-A_{x x}\right) / \hbar \sim 5 \times\right.$ $10^{8} \mathrm{~s}^{-1}$ ], and therefore the $94-\mathrm{GHz}$ spectra still retain the sensitivity to molecular mobility that accounts for much of the success of normal spin-label methodology. At much higher fields, such motions may be driven into the conventional slowmotional regime or even to a pseudo-rigid limit, if the increased Zeeman anisotropy is considerably larger than the rotational frequency. In addition, at operating frequencies in the $94-\mathrm{GHz}$ region, biological samples in nonfrozen aqueous media are still amenable to study with cavity resonators.

Recently, 94-GHz spectra have been published of spinlabeled lipids in phospholipid membranes containing cholesterol (1) and in those without cholesterol (2). Compared with previous high-field studies that have concentrated mostly on rigid-limit spectra and spin Hamiltonian parameters from frozen samples (3), these spectra are from membranes dispersed in liquid water and are therefore sensitive to the lipid dynamics. Most interestingly, resolution of the $g_{x x}$ and $g_{y y}$ features (i.e., $g_{x x}-g_{y y}$ anisotropy) was found for phospholipid chains spin labeled toward their polar group end in cholesterol-containing membranes in the liquid-ordered phase (1). On the other hand, in gel-phase membranes not containing cholesterol the spin-labeled lipid chains are found to be disordered about their $z$-axis, i.e., almost complete averaging of the $g_{x x}$ and $g_{y y}$ resonances (2), even though the chains are almost fully extended in the gel state. The results from the cholesterol-containing membranes are particularly significant because this liquid-ordered state has been proposed to exist in spatially defined regions or domains of the plasma membrane of epithelial cells, contributing to their detergent insolubility and to the sorting processes taking place in intracellular membrane traffic (4).

In principle, resolution of the $g_{x x}$ and $g_{y y}$ resonances in the high-field spectra may be a result of two different modes of rotational motion. Relatively rapid nonaxial rotation with restricted angular amplitude would lead to a partial averaging of the $g_{x x}-g_{y y}$ tensor anisotropy (i.e., in-plane ordering). Alter- 
natively, slow unrestricted axial spin-label rotation in the $x-$ $y$ plane with a correlation time such that $\tau_{\mathrm{R} \|} \gg \hbar /\left(g_{x x}-\right.$ $\left.g_{y y}\right) \beta H$ could also give rise to incomplete averaging of the $g_{x x}-g_{y y}$ anisotropy. Simulations of the high-field EPR spectra that take account of the anisotropic rotational dynamics are therefore of fundamental importance for the analysis of lipid spin labels in membranes.

In the present paper, we have investigated the sensitivity of 94-GHz spectra to anisotropic molecular ordering and rotational dynamics, with special emphasis on rotation about the spin-label $z$-axis. In particular, we have tried to discriminate between the two limiting types of rotational motion by using two corresponding, relatively simple, simulation models. Our aim in selecting these models has been to limit the number of free simulation parameters to a minimum consistent with adequate differentiation between the two physical cases.

The first model (5), using perturbation theory, is valid for relatively rapid rotational motions that are restricted in angular amplitude of both the off-axis motion $(\theta)$ of the nitroxide $z$-axis and the rotation $(\phi)$ around the $z$-axis (i.e., the $x-y$ amplitude). The second model, which is particularly appropriate to slow rotational motions, involves uncorrelated jumps of the nitroxide $z$-axis within a limited angular range and rotational jumps around the $z$-axis that take place over the full angular range $\phi=0-360^{\circ}(6,7)$. With these two models it is possible to compare the relative effects of axial (i.e., $\phi$ ) rotation of limited amplitude with those of slow axial rotations of larger amplitude. It is found that the fast motional model is capable of describing the experimental spectra with a reasonable degree of precision. The rotational rates obtained with the model that is valid also for slow rotational motion are reasonably consistent with those obtained from the fast motional model, i.e., they lie toward the lower extreme of the fast motional region.

\section{SIMULATION METHODS}

\section{(1) Rapid Motion of Limited $\theta$ and $\phi$ Amplitude}

The fast motional model follows the work of Israelachvili et al. (5). The motionally averaged spectral line positions are determined by the maximum angular excursions, $\theta=\theta_{0}$, of the nitroxide $z$-axis and of the azimuthal excursions, $\phi=\phi_{0}$, of the $x$ - (or $y$-) axis about the $z$-axis in the molecular frame. Within these angular ranges, the probability of a particular angular orientation $(\theta, \phi)$ is taken to be random (i.e., the usual $\sin \theta$ weighting for the $\theta$-distribution). The rotational Lorentzian line broadening of the individual hyperfine lines, $m_{l}$, consists of two terms corresponding to axial rotation and off-axial wobbling (cf. Refs. 5, 6). In order to limit the number of adjustable parameters in the simulations, we used the same rotational correlation time for both motions. Then the rotational line broadening is given by

$$
\frac{1}{T_{2, m_{I}}}=\left\langle\left[\omega_{m_{I}}(\theta, \phi)-\left\langle\omega_{m_{I}}\left(\theta_{\mathrm{o}}, \phi_{\mathrm{o}}\right)\right\rangle\right]^{2}\right\rangle_{\theta_{0}, \phi_{\mathrm{o}}} \tau_{\mathrm{R}},
$$

where $\omega_{m_{I}}(\theta, \phi)$ is the instantaneous resonance position and $\left\langle\omega_{m_{I}}\left(\theta_{0}, \phi_{0}\right)\right\rangle$ is its time- (i.e., angular) averaged value. The outer angular brackets indicate the average over the limited orientational range $\theta=0$ to $\theta_{\mathrm{o}}, \phi=0$ to $\phi_{\mathrm{o}}$, and $\tau_{\mathrm{R}}$ is the rotational correlation time. Values of $\omega_{m_{I}}(\theta, \phi)$ and the angular averages are obtained essentially as described in Ref. (5).

\section{(2) Angular Jumps of Limited $\theta$ Amplitude and Unrestricted Frequency}

The anisotropic jump model follows Livshits ( 6 ) and is an extension of the symmetric top model to rotation in an anisotropic medium. The Bloch equations for the transverse magnetization components $\mathbf{M}(u, \mathrm{v})$ in the rotating frame may be written as (6)

$$
\begin{aligned}
\mathrm{d} \mathbf{M} / \mathrm{d} t & +\left(\mathbf{A}+\tau_{\mathrm{R} \|}^{-1} \mathbf{E}\right) \cdot \mathbf{M} \\
= & \tau_{\mathrm{R} \perp}^{-1} \int_{0}^{\theta_{\mathrm{o}}} \int_{\mathrm{o}}^{2 \pi} \mathbf{M} \cdot \sin \theta \mathrm{d} \theta \mathrm{d} \phi \\
& +\left(\tau_{\mathrm{R} \|}^{-1}-\tau_{\mathrm{R} \perp}^{-1}\right) \int_{\mathrm{o}}^{2 \pi} \mathbf{M} \cdot \mathrm{d} \phi+\mathbf{M}_{\mathrm{o}},
\end{aligned}
$$

where $\mathbf{E}$ is the unit matrix, the static magnetization vector is $\mathbf{M}_{\mathrm{o}}=\left(0, M_{\mathrm{o}}\right)$, and $\tau_{\mathrm{R} \|}^{-1}$ and $\tau_{\mathrm{R} \perp}^{-1}$ are the jump rates for rotation around the $z$-axis and for rotation of the long axis, respectively. The Bloch equation matrix in Eq. [2] is defined by

$$
\mathbf{A}=\left(\begin{array}{cc}
T_{2}^{-1} & \omega-\omega_{\mathrm{o}}(\theta, \phi) \\
-\omega+\omega_{\mathrm{o}}(\theta, \phi) & T_{2}^{-1}
\end{array}\right),
$$

where $\omega$ is the angular frequency of the microwave radiation and $\omega_{0}(\theta, \phi)$ is the Larmor frequency for a general orientation $\theta, \phi$ of the magnetic field. Saturation effects are neglected. In the high-field approximation, the angular-dependent expression for the spin-label Larmor frequency is given by

$$
\omega_{\mathrm{o}}(\theta, \phi)=\frac{g_{\mathrm{e}} \omega_{\mathrm{e}}}{g_{Z Z}(\theta, \phi)}-A_{Z Z}(\theta, \phi) m_{I},
$$

where $g_{\mathrm{e}}$ and $\omega_{\mathrm{e}}$ are the free electron $g$-value and Larmor frequency, respectively, and the hyperfine constants are expressed in angular frequency units. The $g_{z z}$ and $A_{z z}$ tensor components in Eq. [4] are defined in the space-fixed magnetic field laboratory frame. They are expressed in terms of the principal components of the $g$ - and $A$-tensors in the magnetic molecular frame by two successive angular transformations. One transformation is from the laboratory frame to the director 
frame in which the director axis coincides with the membrane normal. The other transformation is from the director frame to the molecular frame. It is assumed that for oxazolidine spin labels covalently attached to the lipid chains the $z$-axes of the $A$ - and $g$-tensors coincide with the rotational symmetry axis. The orientation of the molecular frame relative to the director frame is specified by three Euler angles $(\phi, \theta, \varphi)$, where $\phi$ corresponds to axial rotation about the molecular $z$-axis, $\theta$ is the inclination of the molecular $z$-axis to the membrane normal, and $\varphi$ corresponds to an axial rotation in the plane of the membrane. The model described above implies unrestricted uniaxial rotation over $\phi$ with a frequency $\tau_{\mathrm{R} \|}^{-1}$ and limited rotation over the angle $\theta$ from $0^{\circ}$ to $\theta_{\mathrm{o}}$ with frequency $\tau_{\mathrm{R} \perp}^{-1}$. The steady-state solutions $(\mathrm{d} \mathbf{M} / \mathrm{d} t=$ $0)$ of Eq. [2] give the lineshapes $v\left(\omega, \omega_{0}\right)$ of the EPR absorption corresponding to the individual ${ }^{14} \mathrm{~N}$-hyperfine states. Correspondingly, dispersion contributions to the lineshapes are given by the solutions $u\left(\omega, \omega_{\mathrm{o}}\right)$.

A high-field approximation was used for the $g$ - and $A$-tensor anisotropies. It should be noted that, although this is a somewhat severe approximation for EPR at lower static fields, it is much less significant in high-field EPR spectra, where the $g$-tensor anisotropy dominates.

A characteristic feature of the high-field EPR spectra of spin labels is the difference in the linewidth corresponding to the $x$, $y$, and $z$-canonical orientations $(3,8)$. This is assumed to be due to inhomogeneity of the local electric fields, resulting in $g$-strain, which is maximum for the $g_{x x}$-resonance position. Correspondingly, in both models we have introduced different linewidths for the $x, y$, and $z$ orientations in the molecular frame, which were then transformed into the laboratory frame in the same manner as described for the $g$ - and $A$-tensor components.

The CPU time required with a 366-MHz Pentium II computer was typically about $15 \mathrm{~s}$ for simulations with the first model. For the second model, CPU times were in the range of 5-15 min., depending on the intrinsic linewidths and hence on the number of integration points.

\section{RESULTS AND DISCUSSION}

First the sensitivity of the spectral lineshapes to parameters governing the rotational mobility is explored in the two models. The spin Hamiltonian parameters and intrinsic linewidths used for these simulations are those established for experimental spectra. Then simulations are obtained for the experimental spectra of lipids spin labeled at different positions in their fatty acid chains that are incorporated in phospholipid membranes with and without cholesterol.

\section{Model Simulations}

Figure 1 gives simulations using the fast motional formalism for increasing amplitudes $\phi_{\mathrm{o}}$ of axial rotation about a fixed $z$-axis (i.e., $\theta_{\mathrm{o}}=0$ ), at a rate $\tau_{\mathrm{R}}^{-1}=6 \times 10^{8} \mathrm{~s}^{-1}$. Spin
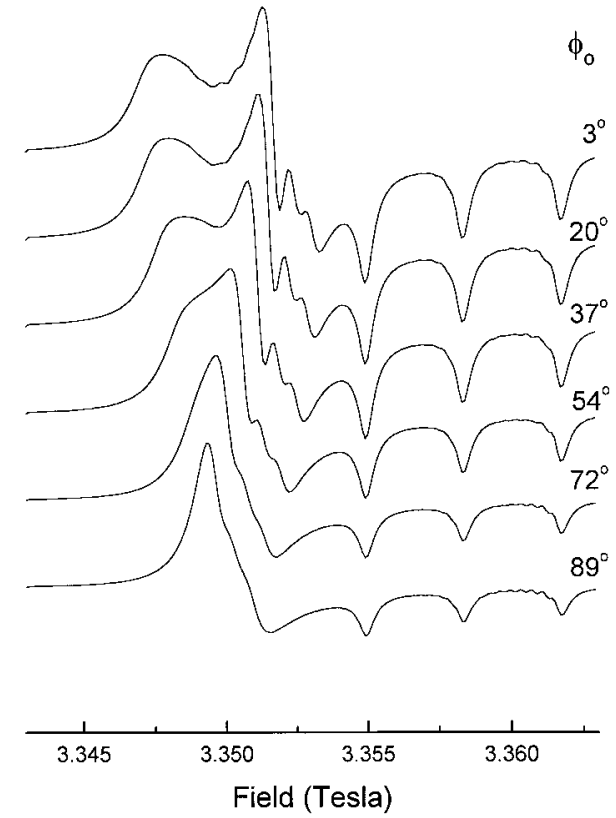

FIG. 1. Simulations of the effect of increasing azimuthal rotational amplitude, $\phi$, on $94-\mathrm{GHz}$ spin-label EPR spectra in the absence of off-axis rotation (i.e., $\theta_{\mathrm{o}}=0$ ) and a fixed rotational rate of $\tau_{\mathrm{R}}^{-1}=6 \times 10^{8} \mathrm{~s}^{-1}$, using the fast motional formalism.

Hamiltonian parameters and intrinsic linewidths used in these simulations are representative of those for experimental spectra of oxazolidine nitroxides (see later). Increasing amplitudes of rotation progressively average the anisotropy between the $g_{x x}$ and $g_{y y}$ canonical regions of the spectrum, whereas the $g_{z z}$ region remains constant. The motionally averaged components in the $g_{x x}, g_{y y}$ region are broadened to an extent that depends on the rotational correlation time and the amplitude of the rotation. Figure 2 shows the dependence of the spectra obtained with the same motional model and increasing amplitude, $\theta_{\mathrm{o}}$, of the off-axis rotation. In this case, full axial rotation (i.e., $\phi_{\mathrm{o}}=360^{\circ}$ ) is assumed and the rate of off-axial rotation is equal to that of the axial rotation: $\tau_{\mathrm{R}}^{-1}=6 \times 10^{8} \mathrm{~s}^{-1}$. At this rotational frequency, the $g_{x x}-g_{y y}$ anisotropy is essentially averaged completely, even with $\theta_{\mathrm{o}}=0$, i.e., in the absence of off-axial motion. For the simulations in Fig. 2, the anisotropy in the $g_{z z}$-region is progressively reduced as the angular amplitude $\theta_{0}$ is increased. Simultaneously, the $x-y$ region moves upfield toward the $z$-region and becomes further broadened. Figure 3 illustrates the corresponding spectral changes when the axial and off-axial amplitudes are increased simultaneously. In this case, the $\left(g_{x x}-g_{y y}\right)$ and $\left(g_{z z}-g_{y y} / g_{x x}\right)$ anisotropies decrease in parallel, which corresponds to the experimentally observed situation (see later, e.g., Fig. 5).

Figure 4 illustrates the alternative situation in which averaging of the spectral anisotropy takes place by an increase in the rate of axial rotation, with fixed amplitudes and rates of off-axis rotation. For this case, the $g_{z z}$-region of the spectrum remains approximately constant, determined by the fixed pa- 


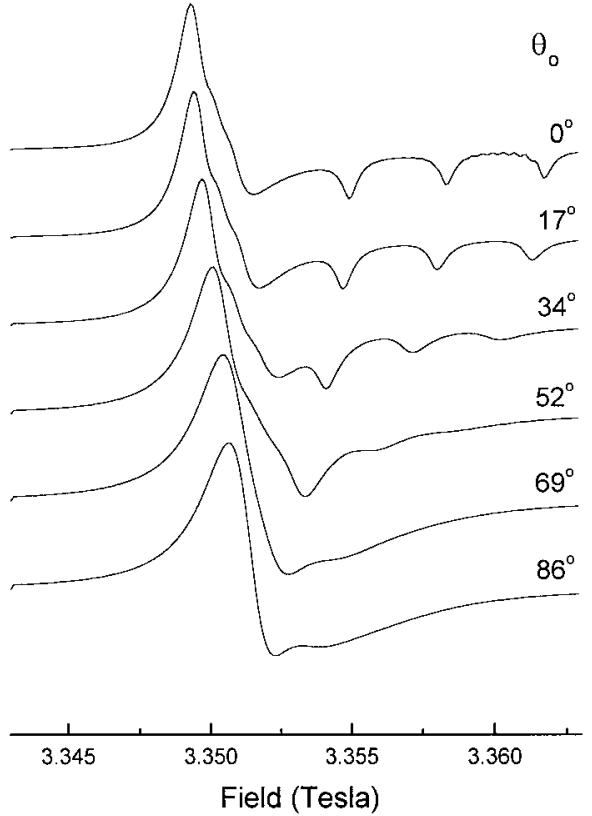

FIG. 2. Simulation of the effect of increasing off-axial rotational amplitude, within a cone of angle $\theta_{\mathrm{o}}$, on $94-\mathrm{GHz}$ spin-label EPR spectra with full axial rotation (i.e., $\phi_{\mathrm{o}}=180^{\circ}$ ) and a fixed rotational rate of $\tau_{\mathrm{R}}^{-1}=6 \times 10^{8} \mathrm{~s}^{-1}$, using the fast motional formalism.

rameters of the off-axial rotation. As the rate of axial rotation increases, the separation of the $g_{x x}$ and $g_{y y}$-features in the spectrum gradually decreases. At sufficiently high axial rotation frequencies, $\tau_{\mathrm{R} \|}^{-1} \sim 0.5-1 \times 10^{9} \mathrm{~s}^{-1}$, the $g_{x x}-g_{y y}$ anisotropy becomes completely averaged (for $\theta_{0}=50^{\circ}$ ). Comparison of Fig. 4 with the preceding figures suggests that the differential patterns of line broadening that accompany loss of the $g_{x x}-g_{y y}$ anisotropy should be sufficient to distinguish between the two models.

\section{Simulation of Experimental Spectra}

A significant problem in recording EPR spectra of aqueous samples at high microwave operating frequencies is the possible admixture of dispersion signals. This is an important technical consideration with membrane samples, the particulate nature of which may require the use of sample tubes with suboptimal diameters, possibly coupled with relatively high electrolyte concentrations. For this reason, a variable admixture of dispersion signal was included in the simulated spectra, at the final stage of the fitting procedure, to check whether this improved the fit. In cases where this was found necessary, the degree of admixture was essentially independent of the fitting parameters.

Spin Hamiltonian tensor values and static linewidths were first determined by simulation of the pseudo-rigid limit spectrum of a dry lipid sample containing a spin-labeled fatty acid (5-(4,4-dimethyloxazolidine- $N$-oxyl)stearic acid; 5-SASL).
The values were then subsequently optimized for the various hydrated samples by small adjustments to allow for the different local environments and polarities. These parameters are collected together in Table 1. Because $g$-tensor components are known accurately for $n$-SASLs only at $77 \mathrm{~K}$ (3), these were maintained constant for the purpose of comparison of the two simulation models (but see later).

Dimyristoyl phosphatidylcholine/cholesterol $(3: 2 \mathrm{~mol} / \mathrm{mol})$ membranes. The 94-GHz spectra of phosphatidylcholine spin labeled on the $n$-C atom of the $s n-2$ chain (1-acyl-2- $[n-(4,4-$ dimethyloxazolidine- $N$-oxyl)stearoyl]-sn-glycero-3-phosphocholine; $n$-PCSL), in fully hydrated membranes of dimyristoyl phosphatidylcholine/cholesterol 3:2 $\mathrm{mol} / \mathrm{mol}$ mixtures (from Ref. 1), are given in Fig. 5. Simulations of the spectra by using the rapid motional model with limited axial rotation (model I) and the model with full axial rotation but general rotational frequency (model II) are given by the dotted and dashed lines, respectively. Also included in the figure is the spectrum of the 5-SASL spin label in a dry mixture of dimyristoyl phosphatidylcholine/myristic acid $1: 2 \mathrm{~mol} / \mathrm{mol}$. It will be noted that simulation of the 8-PCSL spectrum requires a considerable admixture of the dispersion component. The motional parameters that provide the best fitting simulated spectra are given for the two models in Table 2.

As already noted, the dispersion admixture can be determined after optimizing the motional parameters in the simulations. This is illustrated in Fig. 6 for simulation of the spectrum of 8-PCSL in dimyristoyl phosphatidylcholine/cholesterol $(60: 40 \mathrm{~mol} / \mathrm{mol}) \mathrm{membranes}$. By varying the motional simula-

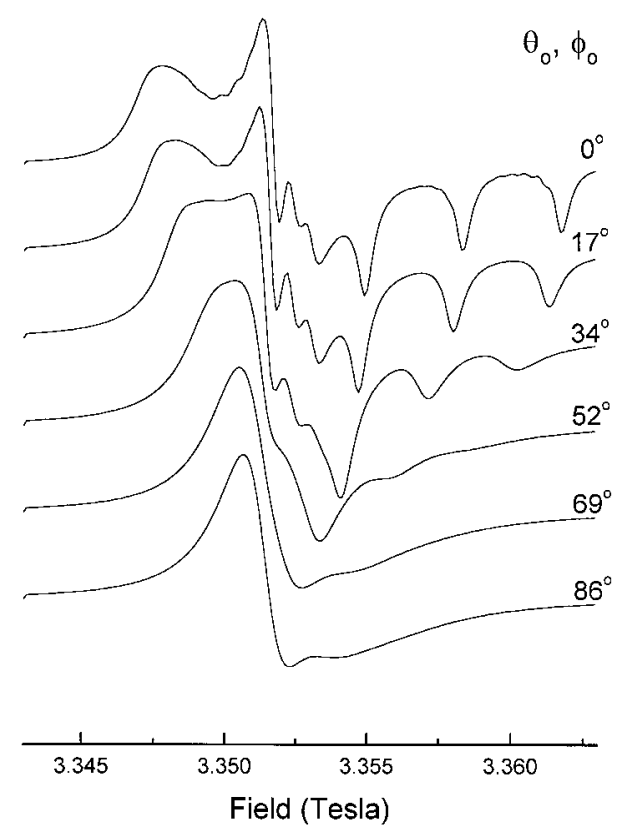

FIG. 3. Simulation of the effect of simultaneously increasing off-axial and azimuthal rotational amplitudes, $\theta_{\mathrm{o}}$ and $\phi_{\mathrm{o}}$, for a fixed rotational rate of $\tau_{\mathrm{R}}^{-1}=$ $6 \times 10^{8} \mathrm{~s}^{-1}$, using the fast motional formalism. 

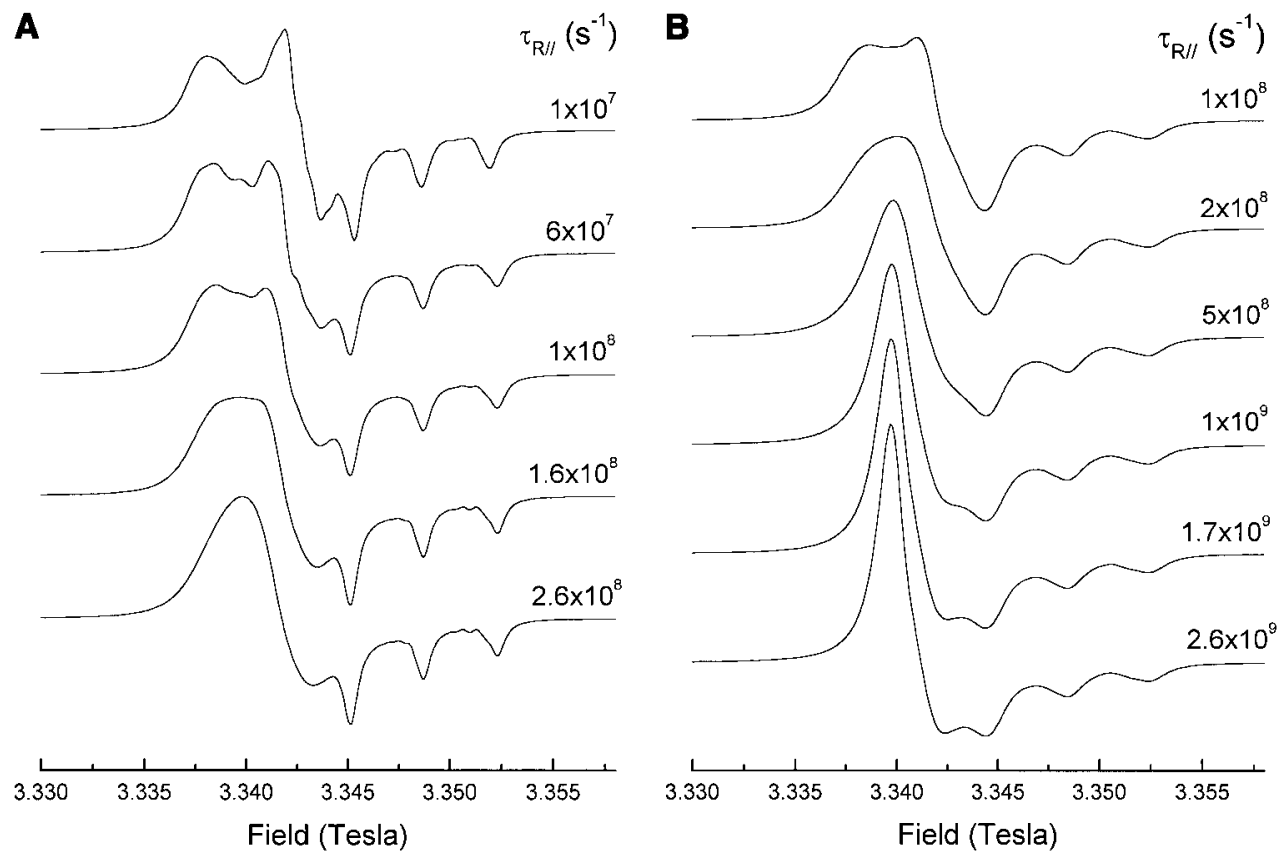

FIG. 4. Simulation of the effect of increasing rate of axial (i.e., $\phi$ ) rotation on the 94-GHz spin-label EPR spectra, for a fixed off-axis rotational amplitude within a cone of angle (A) $\theta_{\mathrm{o}}=30^{\circ}$ and (B) $\theta_{\mathrm{o}}=50^{\circ}$ and a fixed overall rotational rate of (A) $\tau_{\mathrm{R}}^{-1}=10^{7} \mathrm{~s}^{-1}$ and (B) $\tau_{\mathrm{R}}^{-1}=10^{8} \mathrm{~s}^{-1}$, using the strong jump model.

tion parameters alone, without dispersion admixture, it is not possible to reproduce the characteristic baseline distortions of the first-derivative experimental spectrum. Also, the negative excursions of the corresponding integrated experimental spectrum are not generated by the motional model. Increasing admixture of the dispersion component to the simulated ab- sorption spectrum progressively changes the relative amplitudes of the first-derivative peaks and produces the baseline distortions evident in the experimental spectrum, without changing the peak positions or widths appreciably (see Fig. 6). The latter is in contrast to the effects of varying the motional parameters (cf. Figs. 1-3). As an independent criterion for the

TABLE 1

Hyperfine Tensor Elements $A_{i i}$ (in mT) and Static Linewidths $\Delta H_{i}$ (in mT) U sed for Simulation of the 94-GHz EPR Spectra of $n$-PCSL and $n$-SASL Spin Labels in the Various Membrane Samples ${ }^{a}$

\begin{tabular}{|c|c|c|c|c|c|c|c|}
\hline Spin label & $T\left({ }^{\circ} \mathrm{C}\right)$ & $\Delta H_{x}$ & $\Delta H_{y}$ & $\Delta H_{z}$ & $A_{x x}$ & $A_{y y}$ & $A_{z z}$ \\
\hline \multicolumn{8}{|c|}{ Dimyristoyl phosphatidylcholine/myristic acid $(1: 2 \mathrm{~mol} / \mathrm{mol})^{b}$} \\
\hline 5-SASL & 22 & 0.85 & 0.4 & 0.4 & 0.65 & 0.55 & 3.39 \\
\hline \multicolumn{8}{|c|}{ Dimyristoyl phosphatidylcholine/cholesterol $(3: 2 \mathrm{~mol} / \mathrm{mol})$} \\
\hline 8-PCSL & 22 & 0.85 & 0.4 & 0.4 & 0.65 & 0.52 & 3.37 \\
\hline 12-PCSL & 22 & 0.8 & 0.4 & 0.4 & 0.65 & 0.50 & 3.35 \\
\hline 14-PCSL & 22 & 0.8 & 0.4 & 0.4 & 0.63 & 0.50 & 3.08 \\
\hline \multicolumn{8}{|c|}{ Dimyristoyl phosphatidylcholine } \\
\hline \multirow[t]{3}{*}{ 5-SASL } & 21 & 0.85 & 0.4 & 0.4 & 0.65 & 0.50 & 3.45 \\
\hline & 31 & 0.4 & 0.4 & 0.4 & 0.65 & 0.50 & 3.45 \\
\hline & 41 & 0.3 & 0.3 & 0.3 & 0.65 & 0.50 & 3.41 \\
\hline \multirow[t]{3}{*}{ 12-SASL } & 21 & 0.85 & 0.4 & 0.4 & 0.60 & 0.55 & 3.18 \\
\hline & 31 & 0.40 & 0.40 & 0.40 & 0.55 & 0.55 & 3.18 \\
\hline & 41 & 0.40 & 0.35 & 0.4 & 0.55 & 0.55 & 3.18 \\
\hline \multirow[t]{3}{*}{ 16-SASL } & 21 & 0.65 & 0.35 & 0.35 & 0.60 & 0.50 & 3.18 \\
\hline & 31 & 0.35 & 0.35 & 0.35 & 0.60 & 0.50 & 3.18 \\
\hline & 41 & 0.325 & 0.325 & 0.325 & 0.60 & 0.50 & 3.18 \\
\hline
\end{tabular}

${ }^{a}$ A fixed $g$-tensor with elements $\mathbf{g}=(2.00895,2.00628,2.00263)$ was used for all simulations.

${ }^{b}$ Dry sample; all others are hydrated lipid dispersions. 


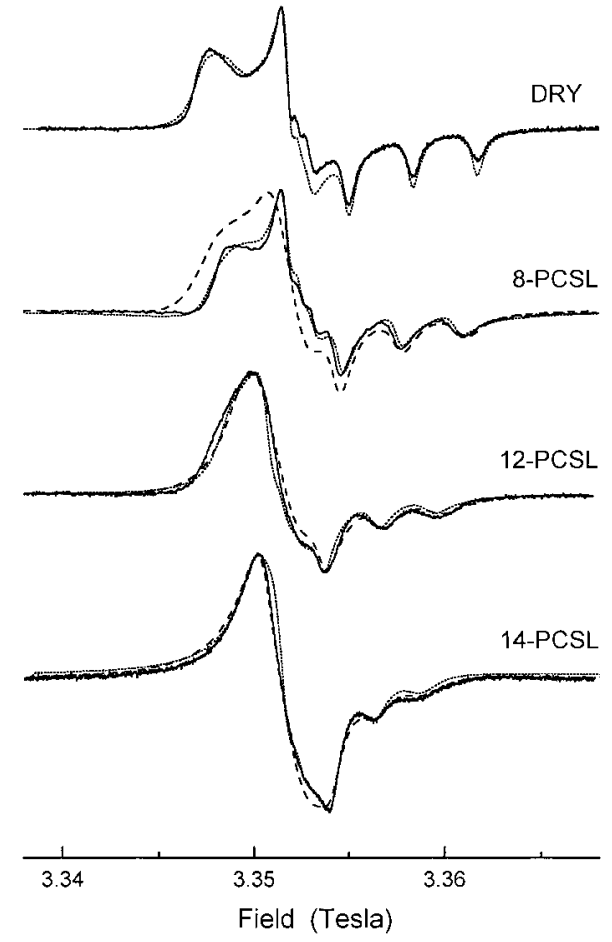

FIG. 5. Simulations of experimental $94-\mathrm{GHz}$ spectra from $n$-PCSL spin labels $(n=8,12,14)$ in dimyristoyl phosphatidylcholine/cholesterol $(60: 40$ $\mathrm{mol} / \mathrm{mol}$ ) membranes (four lower spectra). Full lines, experimental spectra (1); dotted lines, simulations with the fast motional model; dashed lines, simulations with the strong jump model. Dynamic parameters used in the simulations are given in Table 2, with the spin Hamiltonian parameters and intrinsic linewidths given in Table 1. The topmost spectrum is from 5-SASL in a dry sample of DMPC/myristic acid 1:2 $\mathrm{mol} / \mathrm{mol}$, simulated as a rigid limit spectrum with spin Hamiltonian parameters given in Table 1 .

amount of dispersion admixture, the lineshape of the first integral of the spectrum was also used.

The results in Table 2 describe the increasing averaging of the $\left(g_{x x}-g_{y y}\right)$ anisotropy on proceeding down the phospho-

TABLE 2

Angular Amplitudes, $\theta_{0}$ and $\phi_{0}$, and R otational Frequencies, $\tau_{R}^{-1}$, $U$ sed in Simulation of the 94-GHz EPR Spectra of $n$-PCSL Phosphatidylcholine Spin Labels in Hydrated Membranes of Dimyristoyl Phosphatidylcholine/cholesterol 3:2 $\mathrm{mol} / \mathrm{mol}$ Lipid Mixtures

\begin{tabular}{lrrrr}
\hline Spin label & Model $^{a}$ & $\theta_{\mathrm{o}}$ & $\phi_{\mathrm{o}}$ & $\tau_{\mathrm{R}}^{-1}\left(\mathrm{~s}^{-1}\right)$ \\
\hline 8-PCSL & I & $25^{\circ}$ & $26^{\circ}$ & $6 \times 10^{8}$ \\
& II & $26^{\circ}$ & $180^{\circ}$ & $1.26 \times 10^{8}$ \\
12-PCSL & I & $37^{\circ}$ & $57^{\circ}$ & $8 \times 10^{8}$ \\
& II & $37^{\circ}$ & $180^{\circ}$ & $5.05 \times 10^{8}$ \\
14-PCSL & I & $48^{\circ}$ & $66^{\circ}$ & $10^{9}$ \\
& II & $48^{\circ}$ & $180^{\circ}$ & $6.5 \times 10^{8}$ \\
\hline
\end{tabular}

\footnotetext{
${ }^{a}$ Model I: rapid anisotropic rotation of limited angular amplitudes $\theta_{0}$ and $\phi_{0}$. Model II: anisotropic jump rotation of general rate and unrestricted azimuthal amplitude $\left(\phi_{\mathrm{o}}=180^{\circ}\right)$.
}

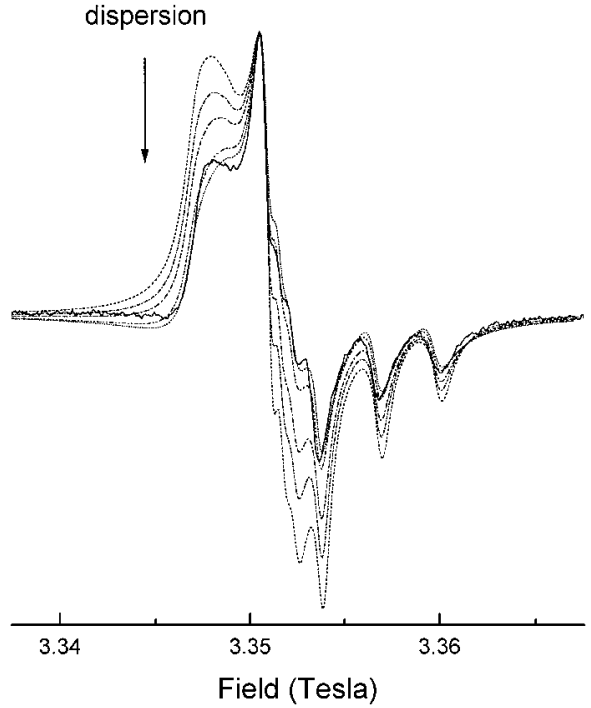

FIG. 6. Effect of increasing dispersion admixture on $94-\mathrm{GHz}$ spectra simulated for 8-PCSL in dimyristoyl phosphatidylcholine/cholesterol (60:40 $\mathrm{mol} / \mathrm{mol}$ ) membranes with the fast motional model. The simulated spectrum of greatest amplitude in the low-field region (short dashes) is the pure firstderivative absorption with the motional parameters given in Table 2. Decreasing amplitude in the low-field region of the simulated spectra represents increasing dispersion admixture.

lipid chain from 8-PCSL to 14-PCSL either in terms of an increasing azimuthal amplitude of motion, $\phi_{0}$ (model I), or in terms of an increasing rate $\tau_{\mathrm{R}}^{-1}$ of unrestricted azimuthal rotation (model II). However, Fig. 5 shows that in general more satisfactory fits to the experimental spectra are obtained with model I. This is particularly the case for the spectrum of 8-PCSL which displays the largest residual anisotropy. For model I, the fits of the simulations to the experimental spectra are reasonably adequate for all positions of chain spin labeling. Both models describe the simultaneous averaging of the $\left(g_{z z}-\right.$ $\left.g_{y y} / g_{x x}\right)$ anisotropy in terms of an increasing amplitude, $\theta_{0}$, of off-axial rotation. However, for matching the experimental splittings, model II results in an increase in relative intensity of the $g_{x x}$-resonance and a considerable broadening at the $g_{x x}{ }^{-}$, $g_{y y^{-}}$, and $g_{z z}$-positions, relative to the spectrum observed experimentally.

A modification of model II was investigated in which the off-axis motion was restricted by a continuous orienting potential, rather than by a fixed cone angle. The best fits with the orienting potential were qualitatively similar to those for the cone model. The excessive broadening of the $g_{x x}, g_{y y}$,- and $g_{z z}$-resonances for 8-PCSL still remained. Line narrowing by increase in the rotational frequency of model II did not result in better fits. The resulting increased axial averaging of the anisotropy in the resonance positions was then greater than that observed experimentally. In addition, simulations were also performed with unequal rates of axial rotation and off-axis wobbling for model II (i.e., $\tau_{\mathrm{R} \|}^{-1} \neq \tau_{\mathrm{R} \perp}^{-1}$ in Eq. [2]). The rms deviation from the experimental spectrum was not improved, 
TABLE 3

Angular Amplitudes, $\theta_{0}$ and $\phi_{0}$, and Rotational Frequencies, $\tau_{\mathrm{R}}^{-1}$, U sed in Simulation of the 94-GHz EPR Spectra of $n$-SASL Spin Labels in Hydrated Dimyristoyl Phosphatidylcholine Membranes

\begin{tabular}{|c|c|c|c|c|c|}
\hline Spin label & $T\left({ }^{\circ} \mathrm{C}\right)$ & Model $^{a}$ & $\theta_{\mathrm{o}}$ & $\phi_{\mathrm{o}}$ & $\tau_{\mathrm{R}}^{-1}\left(\mathrm{~s}^{-1}\right)$ \\
\hline \multirow[t]{6}{*}{ 5-SASL } & \multirow[t]{2}{*}{21} & I & $43^{\circ}$ & $68^{\circ}$ & $1.0 \times 10^{9}$ \\
\hline & & II & $41^{\circ}$ & $180^{\circ}$ & $1.5 \times 10^{9}$ \\
\hline & \multirow[t]{2}{*}{31} & $\mathrm{I}$ & $47^{\circ}$ & $180^{\circ}$ & $1.15 \times 10^{9}$ \\
\hline & & II & $47^{\circ}$ & $180^{\circ}$ & $1.6 \times 10^{9}$ \\
\hline & \multirow[t]{2}{*}{41} & $\mathrm{I}$ & $49^{\circ}$ & $180^{\circ}$ & $1.55 \times 10^{9}$ \\
\hline & & II & $49^{\circ}$ & $180^{\circ}$ & $2.5 \times 10^{9}$ \\
\hline \multirow[t]{6}{*}{ 12-SASL } & \multirow[t]{2}{*}{21} & I & $52^{\circ}$ & $61^{\circ}$ & $6.0 \times 10^{8}$ \\
\hline & & II & $52^{\circ}$ & $180^{\circ}$ & $8.0 \times 10^{8}$ \\
\hline & \multirow[t]{2}{*}{31} & $\mathrm{I}$ & $56^{\circ}$ & $73^{\circ}$ & $8.0 \times 10^{8}$ \\
\hline & & II & $56^{\circ}$ & $180^{\circ}$ & $1.0 \times 10^{9}$ \\
\hline & \multirow[t]{2}{*}{41} & I & $76^{\circ}$ & $180^{\circ}$ & $1.45 \times 10^{9}$ \\
\hline & & II & $72^{\circ}$ & $180^{\circ}$ & $1.5 \times 10^{9}$ \\
\hline \multirow[t]{6}{*}{ 16-SASL } & \multirow[t]{2}{*}{21} & I & $57^{\circ}$ & $67^{\circ}$ & $7.5 \times 10^{8}$ \\
\hline & & II & $57^{\circ}$ & $180^{\circ}$ & $1.0 \times 10^{9}$ \\
\hline & \multirow[t]{2}{*}{31} & $\mathrm{I}$ & $77^{\circ}$ & $180^{\circ}$ & $1.6 \times 10^{9}$ \\
\hline & & II & $77^{\circ}$ & $180^{\circ}$ & $1.6 \times 10^{9}$ \\
\hline & \multirow[t]{2}{*}{41} & I & $80^{\circ}$ & $180^{\circ}$ & $2.9 \times 10^{9}$ \\
\hline & & II & $90^{\circ}$ & $180^{\circ}$ & $2.0 \times 10^{9}$ \\
\hline
\end{tabular}

\footnotetext{
${ }^{a}$ Model I: rapid anisotropic rotation of limited angular amplitudes $\theta_{\mathrm{o}}$ and $\phi_{\mathrm{o}}$. Model II: anisotropic jump rotation of general rate and unrestricted azimuthal amplitude $\left(\phi_{\mathrm{o}}=180^{\circ}\right)$.
}

relative to the restriction $\tau_{\mathrm{R} \|}^{-1}=\tau_{\mathrm{R} \perp}^{-1} \equiv \tau_{\mathrm{R}}^{-1}$. Therefore, results of simulation only with the latter assumption are given for the present data sets (Tables 2 and 3 ).

The simulation results therefore suggest that nonaxial rotation (i.e., in-plane ordering) exists for the $n$-PCSL phospholipid chains in the liquid-ordered phase that is induced by cholesterol. This conclusion is based on the better fits obtained with this simulation model, in comparison with model II. The better fits with model I were preserved when the $g$-tensor components were varied over the range $g_{x x}=$ $2.0087-2.0091, g_{y y}=2.0060-2.00628, g_{z z}=2.0022-$ 2.0027 , respectively. It will be noted that the rotational frequencies required by both models are comparable. Simply, less satisfactory fits to the experimental spectra were obtained with the assumption of unrestricted axial rotation. The relevant rates are within the fast motional regime, for which the spectra are relatively insensitive to the type of rotational motion, e.g., jump vs Brownian diffusion (see, e.g., Ref. 9). However, it has been found by both NMR $(10,11)$ and quasi-elastic neutron scattering (12) that models of restricted and jump rotation adequately describe the in-plane motion of cholesterol. Taking into account the current results, it seems highly plausible that the anisotropic shape of the cholesterol molecule not only results in its restricted and jump-like motion, but also restricts the axial rotation of the neighboring phospholipid molecules, in a similar manner.
Dimyristoyl phosphatidylcholine membranes. The $94-\mathrm{GHz}$ spectra of stearic acid spin labeled on the $n$-C atom of the chain ( $n$-SASL) in fully hydrated bilayer membranes of dimyristoyl phosphatidylcholine (DMPC, from Ref. 2) are given in Fig. 7. At a temperature of $21^{\circ} \mathrm{C}$ hydrated bilayers of this lipid are in the gel phase, and at $31^{\circ} \mathrm{C}$ and above they are in the fluid phase. Simulations of the spectra using the two motional models are given by dotted lines (model I) and by dashed lines (model II). The motional parameters that provide the best fitting simulated spectra are given for the two models in Table 3.

In the gel phase at $21^{\circ} \mathrm{C}$, both models provide a reasonably adequate description of the experimental spectra for the three different positions of chain labeling (Fig. 7). Correspondingly, the motional parameters for the two models are reasonably consistent: a large amplitude of azimuthal rotation and similar rates of rotation (Table 3). Model II provides slightly less good agreement than model I for 12-SASL and 16-SASL, but the values of $\phi_{0}$ required for the latter are uniformly large, so the degree of discrimination is not expected to be very high (cf. Fig. 1). Consistent values of the amplitude, $\theta_{0}$, of off-axial rotation, which increase from 5-SASL to 16-SASL, are obtained for both models.

In the fluid phase, model I gives satisfactory fits for all spin labels, in terms of complete axial rotation, i.e., $\phi_{\mathrm{o}}=180^{\circ}$. An exception is the 12 -SASL spin label at $31^{\circ} \mathrm{C}$, for which the amplitude of axial rotation was estimated to be $\phi_{0} \sim 75^{\circ}$. Both the amplitude, $\theta_{\mathrm{o}}$, and the frequency, $\tau_{\mathrm{R}}^{-1}$, of off-axial rotation increase with increasing temperature in the fluid phase. At a fixed temperature, both also increase from 5-SASL to 16SASL.

In the fluid phase (as in the gel phase), although both models give a moderately adequate representation of the experimental spectra, somewhat better agreement is obtained for model I than for model II (Fig. 7). Furthermore, the rotational frequencies obtained from simulations by using both models are, in general, close to one another (Table 3). Therefore the relatively minor differences between the two sets of simulations are probably due to the different expressions used for the resonance field. In model I, the tensor components are averaged over a cone of angle of $\theta_{0}$, whereas in model II initial nonaveraged tensor components are used that are then averaged explicitly by the rotational motion.

It is to be expected that the behavior of the phosphatidylcholine spin labels, $n$-PCSL, is similar to that of the corresponding stearic acid spin labels, $n$-SASL, in membranes of DMPC alone. Experiments with 5-PCSL in DMPC membranes support this expectation. At low temperature, there is some slight increase in nonaxiality relative to 5-SASL, but this is much smaller than the effects of cholesterol. This nonaxiality disappears at higher temperatures (which is not the case with cholesterol) and the 5-PCSL spectra are very similar to those of 5-SASL given in Fig. 7A.

Compared with the membranes composed of DMPC alone (Table 3), the nonaxial ordering of the lipid chains produced by 


\section{A}

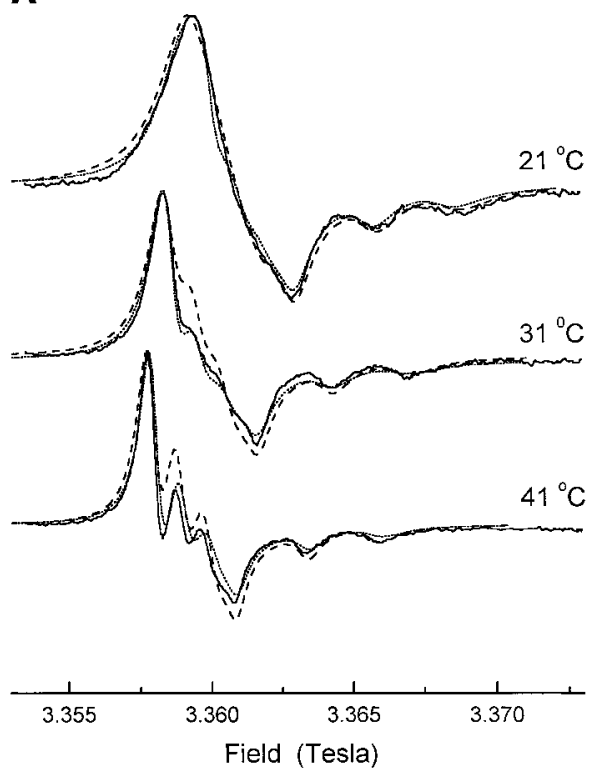

C

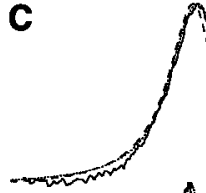

B

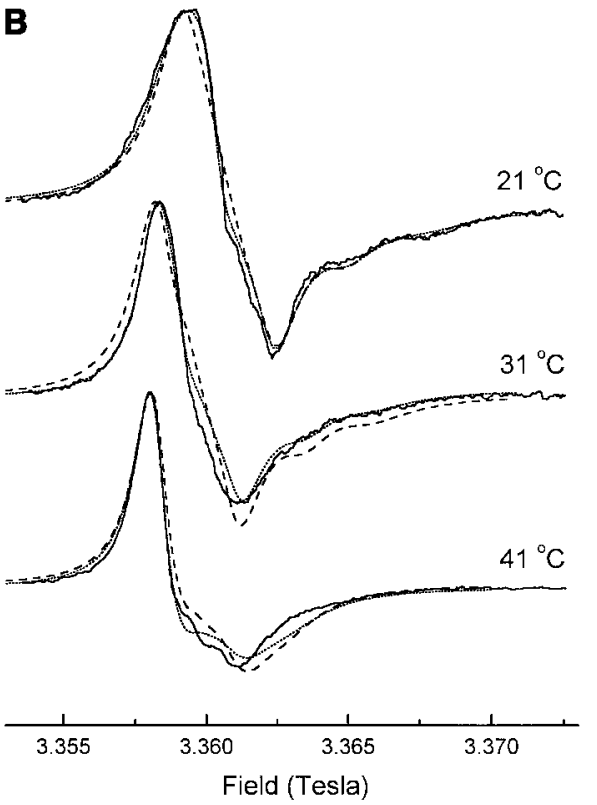

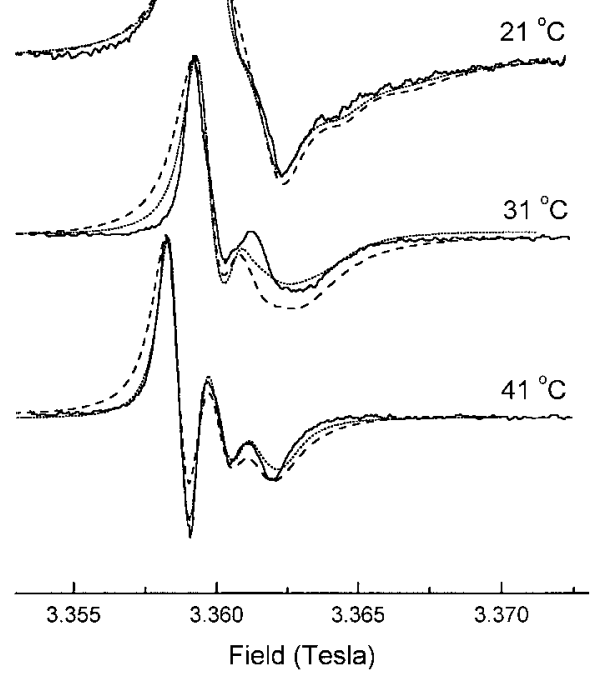

FIG. 7. Simulations of experimental $94-\mathrm{GHz}$ spectra from $n$-SASL spin labels in dimyristoyl phosphatidylcholine membranes at the temperatures indicated. Full lines, experimental spectra (2); dotted lines, simulations with the fast motional model; dashed lines, simulations with the strong jump model. (A) 5-SASL spin label, (B) 12-SASL spin label, and (C) 16-SASL spin label.

cholesterol in DMPC-cholesterol membranes in the liquidordered phase (Table 2) is therefore very pronounced. Even in the gel phase of DMPC membranes, the degree of nonaxial ordering is relatively slight and disappears completely in the fluid phase. This is especially the case in chain regions corresponding to the position of the rigid steroid nucleus of cholesterol (compare 8-PCSL with 5-SASL in Tables 2 and 3, respectively). The possible implications for the formation of sphingolipid-cholesterol domains in natural membranes (4), where the chain-melting transition temperature of the sphingo- lipid component is intrinsically rather high, have already been pointed out (1).

\section{CONCLUSIONS}

The relatively simple methods used for simulation of the two limiting cases of axial rotation have met with reasonable success in defining the origin of the nonaxial anisotropy in the spectra from cholesterol-containing systems. Cholesterol causes in-plane ordering of the lipid chains rather than slowing their axial rotation 
appreciably. The rotational rates are found to be in a time regime for which the spectra are not very sensitive to the mechanism of rotational diffusion. Therefore the computationally simpler and faster noncorrelated jump model is acceptable here and serves to justify use of the analytical perturbation expressions for axial rotation of limited amplitude. Future applications most probably will require also the extension to include Brownian rotational diffusion, which is computationally more demanding. Finally, from a technical point of view, simulation appears to be a viable means for dealing with dispersion admixtures when these technically are not readily avoidable.

\section{ACKNOWLEDGMENT}

This work was supported by the Deutsche Forschungsgemeinschaft.

\section{REFERENCES}

1. B. J. Gaffney and D. Marsh, High-frequency, spin-label EPR of nonaxial lipid ordering and motion in cholesterol-containing membranes. Proc. Natl. Acad. Sci. USA 95, 12940-12943 (1998).

2. A. I. Smirnov, R. L. Belford, and R. B. Clarkson, Comparative spin label spectra at X-band and W-band, in "Biological Magnetic Resonance, Vol. 14, Spin Labelling. The Next Millenium" (L. J . Berliner, Ed.), pp. 83-107, Plenum, New York, 1998.

3. K. A. Earle, J. K. Moscicki, M. Ge, D. E. Budil, and J. H. Freed, $250-\mathrm{GHz}$ electron-spin-resonance studies of polarity gradients along the aliphatic chains in phospholipid-membranes. Biophys. J . 66, 1213-1221 (1994).

4. K. Simons and E. Ikonen, Functional rafts in cell membranes. Nature 387, 569-572 (1997).
5. J. Israelachvili, J. Sjösten, L. E. G. Eriksson, M. Ehrström, A. Gräslund, and A. Ehrenberg, ESR spectral analysis of the molecular motion of spin labels in lipid bilayers and membranes based on a model in terms of two angular motional parameters and rotational correlation times, Biochim. Biophys. Acta 382, 125-141 (1975).

6. V. A. Livshits, Slow anisotropic tumbling in ESR spectra of nitroxyl radicals, J. Magn. Reson. 23, 307-313 (1976).

7. V. A. Livshits, T. Páli, and D. Marsh, Relaxation time determinations by progressive saturation EPR: Effects of molecular motion and Zeeman modulation for spin labels, J. Magn. Reson. 133, 79-91 (1998).

8. M. A. Ondar, O. Ya. Grinberg, A. A. Dubinskii, and Ya. S. Lebedev, Study of the effect of the medium on the magnetic-resonance parameters of nitroxyl radicals by high-resolution EPR spectroscopy, Sov. J . Chem. Phys. 3, 781-792 (1985).

9. J.H. Freed, Theory of slow tumbling ESR spectra of nitroxides, in "Spin Labelling. Theory and Applications" (L. J . Berliner, Ed.), pp. 53-132, Academic Press, New York, 1976.

10. K. Weisz, G. Grobner, C. Mayer, J. Stohrer, and G. Kothe, Deuteron nuclear-magnetic-resonance study of the dynamic organization of phospholipid cholesterol bilayer-membranesMolecular properties and viscoelastic behavior, Biochemistry 31, 1100-1112 (1992).

11. J. M. Bonmatin, I. C. P. Smith, H. C. J arrell, and D. J . Siminovitch, Use of a comprehensive approach to molecular-dynamics in ordered lipid systems-Cholesterol reorientation in oriented lipid bilayers $-A{ }^{2}$ H NMR relaxation case study, J. Am. Chem. Soc. 112, 1697-1704 (1990).

12. C. Gliss, O. Randel, H. Casalta, E. Sackmann, R. Zorn, and T. Bayerl, Anisotropic motion of cholesterol in oriented DPPC bilayers studied by quasielastic neutron scattering: The liquid-ordered phase, Biophys. J . 77, 331-340 (1999). 